\title{
Trocar-guided polypropylene mesh for pelvic organ prolapse surgery-perioperative morbidity and short-term outcome of the first 100 patients
}

\author{
Pia Heinonen • Seija Ala-Nissilä • Riikka Aaltonen • \\ Pentti Kiilholma
}

Received: 18 June 2010 / Accepted: 30 August 2010/Published online: 29 September 2010

(C) Springer-Verlag 2010

\begin{abstract}
This study was conducted to assess the subjective outcome, complications and cure rates of prolapse surgery with a standardized trocar-quided polypropylene mesh in the first 100 patients. A follow-up visit was made after 2 months after the operation and the subjective outcome was assessed with a postal questionnaire 1 year postoperatively. An anterior mesh was used in 48, posterior mesh in 45 , total mesh in five and combined anterior and posterior mesh in two patients. All patients had one or more subjective symptoms. Forty-seven percent of the patients had undergone prolapse surgery and $16 \%$ an antiincontinence operation previously. Two patients had peroperative bleeding of more than $1,000 \mathrm{ml}$, antibiotic treatment was needed in 28 patients and two hematomas were evacuated. A total of 16 patients underwent an antiincontinence operation for de novo stress urinary incontinence. Four patients needed cystocele repair after a posterior mesh and eight patients posterior repair after an anterior mesh. The mesh exposure was diagnosed in 14 patients. No serious complications occurred. Fifty-three $(60 \%)$ patients reported all preoperative symptoms cured, $27(30 \%)$ reported persistent symptoms and five patients were hesitant. Of the respondents, 63 (71\%) were satisfied with the operation. We found that the mesh procedures were associated with a quite high amount of minor postoperative problems.
\end{abstract}

Keywords Pelvic organ prolapse - Polypropylene mesh . Prolift $^{\mathrm{TM}}$. Subjective outcome

P. Heinonen $(\bowtie) \cdot S$. Ala-Nissilä $\cdot$ R. Aaltonen $\cdot$ P. Kiilholma Department of Obstetrics and Gynecology, University of Turku, 20520 Turku, Finland

e-mail: pia.heinonen@tyks.fi

\begin{abstract}
Abbreviations
POP pelvic organ prolapse

SUI stress urinary incontinence

TVT tension-free vaginal tape

TOT transobturator tape
\end{abstract}

\section{Introduction}

Pelvic organ prolapse (POP) has a significant impact on the quality of life of women. Depending on the prolapsed compartment, symptoms may include urinary incontinence, voiding dysfunction, feeling of bulge, difficulty to defecate or fecal incontinence and sexual dysfunction [1]. Symptom relief is an obvious and important outcome measure for patients with pelvic organ dysfunction, but still most studies on POP surgery tend to report objective or anatomical cure as the primary outcome [1].

POP is the most common indication for benign gynecologic surgery and often occurs together with stress urinary incontinence (SUI). Patients' knowledge about urinary incontinence increased substantially over the last decade while knowledge about POP lags behind [1]. Many women treat their symptoms conservatively and only those who experience profound impairment seek surgical solutions. The real incidence of POP is, consequently, unknown but is estimated to vary between $38 \%$ and $41 \%$ of the female population $[2,3]$. The lifetime risk for undergoing an operation for prolapse or urinary incontinence by age of 80 years is $11.1-11.8 \%[4,5]$.

In traditional POP surgery using only native inherently weak or damaged supportive tissues in the pelvic floor, the recurrence rate is no less than $17-29 \%[4,6]$. The development of new surgical techniques with meshes and 
biomaterial implants to reinforce the vaginal wall support has improved results especially by patients undergoing anterior repair, where the failure rate after traditional repair is as high as $40 \%$ [7]. Reconstructions using meshes are efficient $[8,9]$ and overall anatomical cure rates of up to $79-86 \% 1$ year after POP surgery have been reported [10].

New techniques must be evaluated for safety as well as effectiveness. The serious perioperative complication rates related to procedures using meshes in vaginal surgery have been low [11, 12]. However, postoperative mesh-related adverse events, e.g., mesh erosion, may affect the quality of life in the long-term follow-up.

In this study, we have evaluated the subjective outcome and patient satisfaction after pelvic prolapse repair with a standardized trocar-quided polypropylene mesh. The data includes the learning curves of four senior urogynecologists. Complications and any reoperations were reported during follow-up for 1 year. A specific aim was to examine the patient-related outcomes of this technique.

\section{Materials and methods}

This is a clinical follow-up study covering the first 100 patients operated on in the Turku University Central Hospital between June 2005 and April 2007 with the use of a transvaginal polypropylene mesh (Prolift ${ }^{\mathrm{TM}}$, Ethicon, Sommerville, NJ, USA). All operations were performed by four senior urogynecologists.

The data was collected from the hospital records from the preoperative visit to the follow-up visit 2 months after the operation. After an average of 1 year after the operation, a questionnaire was sent to all patients enquiring for the subjective outcome, pelvic floor symptoms and sexual function. The questionnaire was designed by the investigators, since there was no disease-specific, validated quality-of-life questionnaire on functional pelvic floor symptoms available in Finnish. The questionnaire included a detailed list of symptoms (Appendix). Formal, informed consent was obtained from all the patients.

The indication for using mesh was recurrent vaginal prolapse or large primary POP with a paravaginal tissue defect. Validated POP quantification was not used. Of the 100 patients, 49 used vaginal estrogen treatment and 30 had systemic hormone replacement therapy. All patients underwent a clinical examination to assess the site and degree of prolapse. In cases with a history of urinary incontinence a cough test was performed. With the patient in supine position a stress test with a $300-\mathrm{ml}$ bladder volume was performed; a positive cough test was considered to indicate SUI. In these patients, an anti-incontinence operation was planned concomitantly. Urinary sampling was performed before and after the operation. Before the operation, a single dose of metronidazole $(500 \mathrm{mg})$ and of cefuroxime $(1.5 \mathrm{~g})$ was given intravenously. There were eight patients who reported penicillin allergy and they received metronidazole $(500 \mathrm{mg})$ and clindamycin $(600 \mathrm{mg})$ intravenously. Prophylaxis against venous thrombosis was used during the hospital stay. If there was bacterial growth in the preoperative urine sample, an appropriate antimicrobial agent was administered. The criteria for wound infection were soreness, redness, exudation and swelling of the wound. Even a mild increase in the CRP level or fever was treated with antibiotics. Urinary retention was diagnosed if the postvoid residual urine volume was more than $150 \mathrm{ml}$.

A polypropylene mesh was used in all operations. The patients were in the lithotomy position during the procedure. Usually spinal anesthesia was used; nine patients required general anesthesia. Prilocaine with epinephrine was infiltrated in the operative site. The procedure was performed as previously described by Fatton and coworkers [13]. The vaginal mucosa was sutured with continuous or separate absorbable stitches. Finally vaginal packing and urinary catheter were inserted and they were removed in the next morning.

Statistical analysis was done with the SAS ${ }^{\circledR}$ System for Windows, version 8.2 (SAS Institute Inc., Cary, NC, USA). The study was approved by the ethics committee of the Hospital District of Southwest Finland.

\section{Results}

Of the 100 patients, an anterior mesh was used in 48, posterior mesh in 45 , total mesh in five patients and combined anterior and posterior mesh in two patients. The baseline characteristics and preoperative symptoms are detailed in Table 1. Previous gynecological operations had

Table 1 Baseline characteristics of the 100 patients operated on using transvaginal mesh

Characteristics

Age, mean (SD)

$65(10)$

BMI, mean (SD)

27 (4)

Parity, mean (SD)

$2.6(1.3)$

Preoperative symptoms, $n^{\mathrm{a}}$

- Feeling of pressure $\quad 56$

- Difficulties in emptying the bladder 46

- Difficulties in defecation 30

- Urinary incontinence $\quad 29$

- Urinary urgency 19

- Anal incontinence $\quad 7$

- Dyspareunia

4

${ }^{a}$ Patients may have had one or more symptoms 
been performed in 72 patients, 47 for POP and 16 for urinary incontinence (Table 2). The operation characteristics and concomitant surgery are presented in Table 3. Of the patients, $32 \%$ underwent some concomitant operation. Two patients who underwent anterior repair had blood loss exceeding 1,000 $\mathrm{ml}$. Concomitant hysterectomy was performed in one of these procedures. No other serious intraoperative complications such as perforation of adjacent organs occurred.

Postoperatively, 28 patients were treated with antibiotics (Table 4). One haematoma was evacuated after posterior repair during hospital stay and one after anterior repair 3 weeks postoperatively. These hematomas were located under the vaginal mucosa and in both patients the mesh remained intact. Urinary retention $(n=5)$ was treated with an indwelling catheter in three patients for 2 days, with a suprapubic catheter in one patient for 10 days and by observation alone for 2 days in one patient. Exposure of the mesh was reported in 14 patients at follow-up visit 2 months after the operation. In nine patients partial resection of the mesh, trimming and resuturation of the vaginal mucosa was performed. In five patients, exposure was treated by vaginal estrogen alone.

De novo SUI occurred in follow-up visit in 20 patients: in 18 patients after anterior repair and in two patients after posterior repair. This required anti-incontinence surgery for 16 patients (retropubic tape in ten and transobturator tape in six), which was performed at a mean period of 8 months after initial surgery. All of these patients became continent. The remaining four patients with de novo SUI were treated conservatively with pelvic floor muscle training. Six patients complained of urinary frequency and four had difficulties in emptying their bladder or rectum. Twelve reoperations due to POP were necessary during the follow-up of 1 year. In four cases with

Table 2 Previous surgery of the 100 patients operated on using transvaginal mesh

\begin{tabular}{ll}
\hline Number of patients with previous operations $^{\mathrm{a}}$ & 72 \\
\hline Previous operations for POP & 47 \\
- Anterior and/or posterior colporrhaphy & 39 \\
- Vaginal hysterectomy & 29 \\
- Sacrospinous ligament fixation & 9 \\
Anti-incontinence operations & 16 \\
- Burch colposuspension & 8 \\
- TVT & 5 \\
- Burch and TVT & 1 \\
- TOT & 1 \\
- Marshall-Marchetti-Kranz operation & 1 \\
Hysterectomy for other indications than POP & 36 \\
\hline
\end{tabular}

${ }^{\text {a }}$ Forty-three patients have had two or more previous gynecological procedures

Table 3 Operation characteristics and concomitant surgery in 100 patients operated on using transvaginal mesh

Characteristics

\begin{tabular}{ll}
\hline Operation time, median (range) & $72 \mathrm{~min}(27-160)$ \\
- Anterior mesh & $88 \mathrm{~min}(27-160)$ \\
- Posterior mesh & $60 \mathrm{~min}(30-83)$ \\
- Total mesh & $110 \mathrm{~min}(72-160)$ \\
- Anterior and posterior mesh & $113 \mathrm{~min}(110-125)$ \\
Blood loss, median (range) & $100 \mathrm{ml}(10-1100)$ \\
- Anterior mesh & $180 \mathrm{ml}(30-110)$ \\
- Posterior mesh & $45 \mathrm{ml}(10-500)$ \\
- Total mesh & $150 \mathrm{ml}(50-550)$ \\
- Anterior and posterior mesh & $350 \mathrm{ml}(250-450)$ \\
Hospital stay, median (range) & $3 \mathrm{days}(0-12)$ \\
Concomitant operations, $n$ & 32 \\
- Traditional anterior or posterior colporrhaphy & 14 \\
- Sacrospinous fixation & 12 \\
- Anti-incontinence operation & 6 \\
- Vaginal hysterectomy & 2 \\
\hline
\end{tabular}

initial posterior mesh an anterior repair was done and in eight cases of anterior mesh a posterior repair was necessary.

A questionnaire after an average of 1 year was responded by 89 patients after one remainder. Fiftythree $(60 \%)$ patients reported that all of their preoperative symptoms had been cured, 27 (30\%) had persistent symptoms and five were uncertain as to the outcome. The most common persistent symptoms reported were urge incontinence $(n=12)$, SUI $(n=8)$ and urinary frequency $(n=6)$. Forty-three patients $(48 \%)$ complained of one or more de novo symptoms: 15 had pain or dyspareunia, nine had lower urinary tract symptoms, nine had bowel symptoms, seven had a feeling of tension and seven sensation of bulge and ten had urinary incontinence. Sixteen (42\%) of the 38 sexually active patients reported dyspareunia postoperatively. In the majority of these

Table 4 Complications related to 100 operations using transvaginal mesh

Complication

Bleeding $>1,000 \mathrm{ml} 2$

Transient urinary retention $\quad 5$

Hematoma 2

Mesh exposure $\quad 14$

Patients with postoperative antibiotic treatment 28

- Bacteriuria 15

- Wound infection 6

- Elevated CRP and/or mild fever 7

De novo SUI 20 
patients dyspareunia was infrequent. Four patients reported it frequently and two at every intercourse. Sixty-three $(71 \%)$ patients were satisfied with the operation and $73(82 \%)$ would recommend the procedure to a friend.

\section{Discussion}

This study reports the data on our first 100 patients with POP operated on using a polypropylene mesh. All complications and subjective symptoms or complaints were carefully registered and reported.

The exposure rate of the mesh was $14 \%$; also small, nonsymptomatic exposures were recorded. In the literature, mesh exposure rates have varied between $7 \%$ and $17 \%[10$, 14-16]. The erosion rate of vaginal meshes may to be related to surgeon's experience. According to a retrospective review [15] the erosion rate decreased from $19 \%$ to $4 \%$ during a mean follow-up time of 29 months. The fact that our study was conducted in the beginning of the learning curves of four doctors in a new surgical technique may thus have affected the rate of exposures.

We had two intraoperative bleeding complications of blood loss of more than $1,000 \mathrm{ml}$. The patients recovered uneventfully and they had no hematomas at operative site. The mean blood loss was fourfold in using anterior or total mesh compared to posterior mesh (Table 3). Other investigators have similar results. Elmer et al. [10] reported a twofold bleeding rate in patients who underwent anterior repair compared to posterior repair. The high rate of recurrence in the anterior compartment and scarring at the operative site after previous repair may complicate surgical preparation of the anterior compartment. Anterior vaginal dissection may also be associated with excessive bleeding due to injury to vascular structures in the paravesical space [11]. In addition, the passage of four supportive arms of the anterior mesh compared with two arms in the posterior procedure may contribute to a relatively greater blood loss related to anterior procedures.

In our study, one-fourth of the patients received antibiotic treatment postoperatively and no serious meshrelated infections occurred. Still, the overall rate of infections is quite high $(28 \%$, Table 4) compared to previous studies, where $8.7-13.6 \%$ of the patients were treated for infections $[11,13,14]$. This refers to our policy to treat also mild postoperative infections in our first mesh patients in order to prevent more serious adverse events. In addition, a routine postoperative urine sampling despite of missing urinary symptoms had led to a treatment of asymptomatic bacteriuria. It is a known fact that patients with asymptomatic bacteriuria should not be treated with antibiotics in order to prevent increasing antimicrobial resistance [17]. Nowadays, after achieving more experience in mesh surgery urinary samples after the operation are taken only of symptomatic patients.

Occult SUI occurs in $11-22 \%$ of patients after POP surgery [18]; in this study, de novo SUI rate was $20 \%$ and most of these patients needed surgery for SUI later on. Six patients underwent concomitantly an anti-incontinence operation (Table 3). The anterior repair with mesh raises the risk for postoperative SUI from $10 \%$ to $23 \%$ compared to traditional colporraphy [14]. Antiincontinence operations in association with vaginal surgery are reportedly an efficient treatment of occult SUI [19], but the risk for urinary retention must be taken into consideration $[20,21]$. Therefore, although routine preoperative stress test might be positive, one must consider carefully whether concomitant SUI surgery is essential. Sufficient preoperative information about occult SUI and the possibility to treat it later by surgery is important notions. However, subsequent surgery for stress incontinence should not be reported as a reoperation related to prolapse repair [1].

The rather high rate of de novo symptoms reported by our patients postoperatively ( $48 \%$ of the respondents) may partially be artificial, since we used a very detailed questionnaire enquiring subjective outcome. Additionally, questionnaires were filled in 1 year postoperatively which may affect the reliability of reporting preoperative symptoms. However, the prevalence of persistent subjective symptoms was comparable to a recent review where urinary symptoms were persistently reported by $22.7 \%$ patients and bowel symptoms by $4.3 \%$ after surgery using a nonabsorbable mesh [22]. Presumably because of persistent or de novo subjective complaints, satisfaction with the operation was reported by $71 \%$ of the respondents. It is increasingly recognized that in POP surgery subjective outcome is a more appropriate measure of efficacy than objective measures [22].

Although the anatomy after the operation was restored at the follow-up visit 2 months after the operation, by the end of the first postoperative year 12 patients had undergone another procedure for POP. After initial anterior prolapse surgery another procedure was performed in the posterior compartment for eight patients. In the remaining four patients anterior prolapse occurred after a posterior mesh procedure. De novo prolapse rate in the non-mesh-treated site in 1 year follow-up is reported to be concerning high $23 \%$ and reoperation rate $4 \%$ [23]. In our study, reoperation rate for prolapse in another compartment was $12 \%$ during 1 year follow-up. The need for another operation for POP during a short-term follow-up might be possibly related to the previous POP surgery but a new procedure at a new site could also be recorded as "primary" surgery [1]. In our 
study, no validated method was used in pre- and postoperative examination but the follow-up was 1 year, which is considered as a minimum adequate period of time to assess the efficacy of prolapse repair [22]. There have been reports of anatomically high success rates when meshes are used $[10-12,16]$ and also significant improvements in the patients' quality of life have been reported [9]. Anatomical cure is naturally a remarkable indicator for outcome, especially when evaluating new surgical techniques for treating POP. However, it is also important to assess the patients' subjective outcome and symptoms and not just the objective outcome as indicator for good achievement. Paradoxically one may consider the anatomical result as a surrogate endpoint, while the real, meaningful endpoint is reflected by patient-related outcomes. Physicians have a tendency to underestimate the complaints of the patients [24, 25], and this makes it even more important that, when evaluating the success of any operation, especially in POP surgery, the patients' quality of life is considered.

In our study, 100 patients underwent POP repair for which a transvaginal polypropylene mesh was used. In our clinic indications for using mesh is a recurrent vaginal prolapse or large primary POP with a paravaginal tissue defect. In this non-controlled study a comparison with the traditional techniques was not possible. Although many patients experienced subjective complaints or some problems such as mesh exposure or minor infections after operation, there were no serious postoperative complications. The results for the very first 100 procedures performed in our hospital were only fair considering the quite high rate of de novo symptoms and prolapses.

Our study represents the situation where new skills are learned and prevailing practice has not yet been composed with a new procedure. Although all the surgeons have studied the mesh technique in theory and also in hands-on training, their inexperience in new surgery must have affected our complication and re-operation rates. Our preliminary results of the subsequent 100 patients operated on by the same procedure reveal a decrease in complication rates. A careful preparation and training before introducing new surgical techniques is essential to improve the results of the surgery. Consequently, it will be necessary to evaluate the objective long-term outcome as well as subjective outcome and satisfaction of the procedure using validated questionnaires.

Acknowledgements Dr Robert Paul reviewed the language of this manuscript.

Conflict of interest Pia Heinonen received a grant from the University of Turku (Finland). She also received money to support travel to the NUGA Congres Stockholm 2008 for the study or otherwhise from Astellas Pharma. Pia Heinonen's travel and accommodation expenses were covered or reimbursed by Bard (Mesh workshop in New York 2008).

\section{Appendix}

Are You using hormonal treatment?

1) Vaginal estrogen

2) Estrogen tablets/plaster/gel

3) Combination treatment (estrogen and progesterone)

4) Hormonal IUD

Are You using some of the following medications?

1) Cortisone orally

2) Insulin or oral treatment for diabetes

3) Diuretics (e.g., for treating hypertension)

4) Antibiotics for recurrent urinary tract infection

What kind of symptoms did You have before the operation for pelvic organ prolapse? Please circle the symptom symptoms and underline the most predominant symptom.

1) Feeling of pressure

2) Difficulties in emptying the bladder

3) Stress urinary incontinence

4) Urgency

5) Frequency

6) Flatus incontinence

7) Fecal incontinence

8) Difficulties in emptying the rectum

9) Pain during sexual intercourse

10) Other kind of pain

Were the symptoms cured after the operation?

1) Yes

2) No, which symptom remained

Have You developed some new symptoms after the operation?

1) Feeling of pressure or bulge

2) Feeling of tension, e.g. when sitting down

3) Stress urinary incontinence

4) Urge incontinence

5) Frequency

6) Difficulties in emptying the bladder

7) pain during urinating

8) Fecal incontinence

9) Pain during emptying the bowel

10) Difficulties in emptying the bowel

11) Pain during sexual intercourse

12) Other kind of pain, what kind of 
Are You sexually active?

1) Yes

2) No

Do You have pain or stinging during sexual intercourse after the operation?

1) $\mathrm{No}$

2) Occasionally

3) Frequently

4) Every time

Do you have other problems during sexual intercourse?

1) Insensibility

2) Narrowness of the vagina

3) Something else

Have You been reoperated or is a new operation scheduled for gynaecological symptom?

1) $\mathrm{No}$

2) Yes, what operation

Are You satisfied with the outcome of the operation?

1) Yes

2) No

3) Hesitate

Would You recommend this procedure to a friend?

1) Yes

2) No

\section{References}

1. Freeman RM (2010) Do we really know the outcomes of prolapse surgery? Maturitas 65:11-14

2. Hendrix SL, Clark A, Nygaard I, Aragaki A, Barnabei V, McTiernan A (2002) Pelvic organ prolapse in the Women's Health Initiative: gravity and gravidity. Am J Obstet Gynecol 186:1160-1166

3. Swift S, Woodman P, O'Boyle A, Kahn M, Valley M, Bland D, Wang W, Schaffer J (2005) Pelvic Organ Support Study (POSST): the distribution, clinical definition, and epidemiologic condition of pelvic organ support defects. Am J Obstet Gynecol 192:795-806

4. Olsen AL, Smith VJ, Bergstrom JO, Colling JC, Clark AL (1997) Epidemiology of surgically managed pelvic organ prolapse and urinary incontinence. Obstet Gynecol 1997;89:501-506

5. Fialkow MF, Newton KM, Lentz GM, Weiss NS (2008) Lifetime risk of surgical management for pelvic organ prolapse or urinary incontinence. Int Urogynecol J 19:437-440

6. Denman MA, Gregory WT, Boyles SH, Smith V, Edwards SR, Clark AL (2008) Reoperation 10 years after surgically managed pelvic organ prolapse and urinary incontinence. Am J Obstet Gynecol 198:555.e1-555.e5

7. Maher C, Baessler K (2006) Surgical management of anterior vaginal wall prolapse: an evidence based literature review. Int Urogynecol J 17:195-201
8. Sand PK, Koduri S, Lobel RW, Winkler HA, Tomezsko J, Culligan PJ, Goldberg R (2001) Prospective randomized trial of polyglactin 910 mesh to prevent recurrence of cystoceles and rectoceles. Am J Obstet Gynecol 184:1357-1362

9. de Tayrac R, Deffieux X, Gervaise A, Chauveaud-Lambling A, Fernandez H (2006) Long-term anatomical and functional assessment of trans-vaginal cystocele repair using a tension-free polypropylene mesh. Int Urogynecol J 17:483-488

10. Elmer C, Altman D, Ellström Eghn M, Axelsen S, Väyrynen T, Falconer C (2009) Trocar-guided transvaginal mesh repair of pelvic organ prolapse. Obstet Gynecol 113:117-126

11. Altman D, Falconer C, for the Nordic Transvaginal Mesh Group (2007) Perioperative morbidity using transvaginal mesh in pelvic organ prolapse repair. Obstet Gynecol 109:303-308

12. de Tayrac R, Devoldere G, Renaudie J, Villard P, Guilbaud O, Eglin G (2007) The French Ugytex Study Group. Prolapse repair by vaginal route using a new protected low-weight polypropylene mesh: 1-year functional and anatomical outcome in a prospective multicentre study. Int Urogynecol J 18:251256

13. Fatton B, Amblard J, Debodinance P, Cosson M, Jacquetin B (2007) Transvaginal repair of genital prolapse: preliminary results of a new tension-free vaginal mesh (Prolift ${ }^{\mathrm{TM}}$ technique) - a case series multicentric study. Int Urogynecol J 18:743-752

14. Hiltunen R, Nieminen K, Takala T, Heiskanen E, Merikari M, Niemi K, Heinonen PK (2007) Low-weight polypropylene mesh for anterior vaginal wall prolapse: a randomised controlled trial. Obstet Gynecol 110:455-462

15. Dwyer PL, O'Reilly BA (2004) Transvaginal repair of anterior and posterior compartment prolapse with Atrium polypropylene mesh. BJOG 111:831-836

16. Milani R, Salvatore S, Soligo M, Pifarotti P, Meschia M, Cortese $M$ (2005) Functional and anatomical outcome of anterior and posterior vaginal prolapse repair with prolene mesh. BJOG 112:107-111

17. Colgan R, Nicolle L, McGlone A, Hooton T (2006) Asymptomatic Bacteriuria in Adults. Am Fam Physician 74:985-990

18. Haessler AL, Lin LL, Ho MH, Betson LH, Bhatia NN (2005) Reevaluating occult incontinence. Curr Opin Obstet Gynecol 17:535-540

19. Meltomaa S, Backman T, Haarala M (2004) Concomitant vaginal surgery did not affect outcome of the tension-free vaginal tape operation during a prospective 3-year follow-up study. J Urol 172: 222-226

20. Fatton B (2009) Is there any evidence to advocate SUI prevention in continent women undergoing prolapse repair? An overview. Int Urogynecol J 20:235-245

21. Partoll LM. Efficacy of tension-free vaginal tape with other pelvic reconstructive surgery (2002) Am J Obstet Gynecol 186:12921295

22. Jia X, Glazener C, Mowatt G, MacLennan G, Bain C, Fraser C, Burr J (2008) Efficacy and safety of using mesh or grafts in surgery for anterior and/or posterior vaginal wall prolapse: systematic review and meta-analysis. BJOG 115:1350-1361

23. Withagen MIJ, Vierhout ME, Milani AL (2010) Does trocarguided tension-free vaginal mesh (Prolift ${ }^{\mathrm{TM}}$ ) repair provoke prolapse of the unaffected compartments? Int Urogynecol J 21:271-278

24. de Boer TA, Gietelink DA, Vierhout ME (2008) Discrepancies between physician interview and a patient self-assessment questionnaire after surgery for pelvic organ prolapse. Int Urogynecol J 19:1349-1352

25. Srikrishna S, Robinson D, Cardozo L, Gonzalez J (2008) Is there a discrepancy between patient and physician quality of life assessment? Int Urogynecol J Pelvic Floor Dysfunct 19:517-520 